\title{
A European multicentre prospective randomized study to assess the use of assisted hatching with a diode laser and the benefit of an immunosuppressive/antibiotic treatment in different patient populations
}

\author{
M.-P.Primi ${ }^{1}$, A.Senn ${ }^{1}$, M.Montag ${ }^{2}$, H.Van der Ven ${ }^{2}$, J.Mandelbaum ${ }^{3}$, A.Veiga ${ }^{4}$, P.Barri ${ }^{4}$ \\ and M.Germond ${ }^{1,5}$
}

\footnotetext{
${ }^{1}$ Reproductive Medicine Unit, Department of Gynaecology and Obstetrics, CHUV, CH-1011 Lausanne, Switzerland, ${ }^{2}$ Department of Gynaecology and Reproductive Medicine, University of Bonn, D-53105 Bonn, Germany, ${ }^{3}$ IVF and Reproductive Biology Laboratory, Necker Hospital, F-75015 Paris, France and ${ }^{4}$ Dexeus Institute, E-08017 Barcelona, Spain

${ }^{5}$ To whom correspondence should be addressed at: Unité de Médecine de la Reproduction, Maternité, CHUV, CH-1011 Lausanne, Switzerland. E-mail: marc.germond@chuv.hospvd.ch
}

BACKGROUND: Assisted hatching (AH) techniques, designed for facilitating the embryo escape out of the zona pellucida (ZP) have been used in IVF centres since 1992. The initial indications for AH were patient's age, ZP thickness, high basal FSH and repeated IVF failures. Several retrospective and prospective studies assessing AH in these indications have given disparate results. Our aims were to evaluate the benefits of AH and immunosuppressive/antibiotic treatment (IA) in patients with either a poor prognosis of success, previous implantation failures or transfers of cryopreserved embryos. METHODS: Four IVF centres allocated 426 patients, randomized for AH and IA, into four groups of AH indications between 1997 and 1999. AH was performed with a diode laser. ZP thickness, opening size and embryo score were recorded. Outcome measures were implantation and delivery rates. RESULTS: Patients coming for a first or third transfer of cryopreserved embryos and poor prognosis patients admitted for a first trial did not benefit from AH. Even patients with repeated implantation failures of fresh embryos did not gain significantly from AH. CONCLUSIONS: Among AH indications, absence of implantation after several transfers of good quality embryos remains the strongest patient selection criterion. Prescription of an immunosuppressive/antibiotic treatment is essential.

Key words: diode laser/hatching/immunosuppression/pregnancy rate/randomized trial

\section{Introduction}

Less than $20 \%$ of unselected human embryos replaced in the uterus after IVF are able to produce viable pregnancies. Several cumulative factors have been considered to lower the implantation rates: poor in vitro culture conditions of cleavage stage embryos (Bongso et al., 1991), a high rate of aneuploidy observed in human oocytes and embryos (Plachot, 1992; Gianaroli et al., 1999), a controversial reduction of endometrial receptivity (Abdalla et al., 1994; Baruffi et al., 2002) and/or an inability of the blastocyst to escape from its zona pellucida (ZP) (Cohen, 1993; De Vos and Van Steirteghem, 2000). Thinning or opening of the ZP using chemical (Cohen et al., 1992; Tao and Tamis, 1997), mechanical (Stein et al., 1995; Chao et al., 1997), enzymatic (Isik et al., 2000), piezo-micromanipulation (Nakayama et al., 1999) or laser (Germond et al., 1999) methods have been performed for over a decade to assist blastocyst hatching and favour its implantation (Cohen, 1991). An infrared laser method allowing a rapid, precise and non-touch microdissection of the ZP protein matrix (Rink et al., 1994, 1996) has progressively replaced the previously described assisted hatching $(\mathrm{AH})$ techniques. Selective AH using the four methods mentioned above was shown to yield similar implantation and pregnancy rates (Balaban et al., 2002).

Thick or hardened ZP (Cohen et al., 1992; De Vos and Van Steirteghem, 2000) are considered as two major causes able to impede the hatching process. However, the indications for $\mathrm{AH}$ remain elusive and several prospective and retrospective studies have failed so far to demonstrate which indications clearly benefit from AH (Germond et al., 2000; Edi-Osagie et al., 2003).

A possible hazard of $\mathrm{AH}$ is that it deprives the embryo of its protective ZP. Immunosuppression by corticosteroids was initially shown to favour implantation of ZP dissected embryos (Cohen et al., 1990). However, when given to patients receiving a transfer of non-micromanipulated 
embryos, the immunosuppressive treatment was found either to have no clinical benefits (Lee et al., 1994) or to significantly enhance implantation (Polak de Fried et al., 1993).

The two aims of the present study, involving four European IVF centres, were to assess the benefit of laser AH in four groups of patients with distinct $\mathrm{AH}$ indications and to value the usefulness of an associated immunosuppressive therapy.

\section{Materials and methods}

The four IVF centres involved were: (i) the Reproductive Medicine Unit in the CHUV of Lausanne, Switzerland; (ii) the Department of Gynaecology and Reproductive Medicine of the University of Bonn, Germany; (iii) the IVF and Reproductive Biology Laboratory of the Necker Hospital in Paris, France; and (iv) the Dexeus Institute in Barcelona, Spain.

\section{Patients}

A pre-study visit took place 2 months before the enrolment in the study, during which the prospective patient was informed of the study's objectives and overall requirements, received an information document and went through a clinical evaluation to ensure conformity with the eligibility criteria. The inclusion criteria were: (i) aged between 20 and 45 years old, (ii) having at least one functional ovary, (iii) having normal FSH (between 3 and $12 \mathrm{IU} / \mathrm{l}$ ) and prolactin $(<30 \mu \mathrm{g} / \mathrm{l})$ levels, (iv) having no clinically significant abnormal findings within 6 months before treatment start, (v) having no pelvic inflammatory disease between the previous assessment and study entry, (vi) having a normal uterine cavity as documented within 5 years prior to treatment assignment by a hysteroscopy, hysterosalpingography or hysterosonography, (vii) being able to communicate well with the investigator and to comply with the requirements of the entire study, and (viii) having given written informed consent prior to treatment with the understanding that consent may be withdrawn by the patient at any time without prejudice.

The pre-study visit included the following: recording of demographic data, medical and medication history, gynaecological and obstetrical history, physical and gynaecological examinations, diagnostic ultrasound, and blood tests [FSH, prolactin (PRL), rubella, hepatitis B and C, HIV].

Depending on their specific $\mathrm{AH}$ indications, eligible patients were allocated by the supervising medical doctor to one of the treatment groups (I, II, III or IV) described below, and randomized at the beginning of the menstrual cycle during which the embryo transfer was going to take place.

Group I: patients who initiated their first transfer cycle of frozenthawed embryos. AH was performed or not according to a randomization list (Figure 1A).

Group II: patients who had failed to conceive in at least two transfer cycles of frozen-thawed embryos and were initiating a third transfer cycle of thawed embryos. AH was performed on all transferred embryos (Figure 1B).

Group III: a selected group of patients who had a poor prognosis of conception (age $>37$ years old or FSH $>10 \mathrm{UI} / \mathrm{l}$ ) for their first transfer of fresh embryos. AH was performed according to a randomization list (Figure 1A).

Group IV: a selected group of patients who had experienced three previous nidation failures of fresh embryos, including each time the transfer of at least two embryos of good quality, and were initiating their fourth transfer cycle of fresh embryos. AH was performed on all transferred embryos (Figure 1B).
A

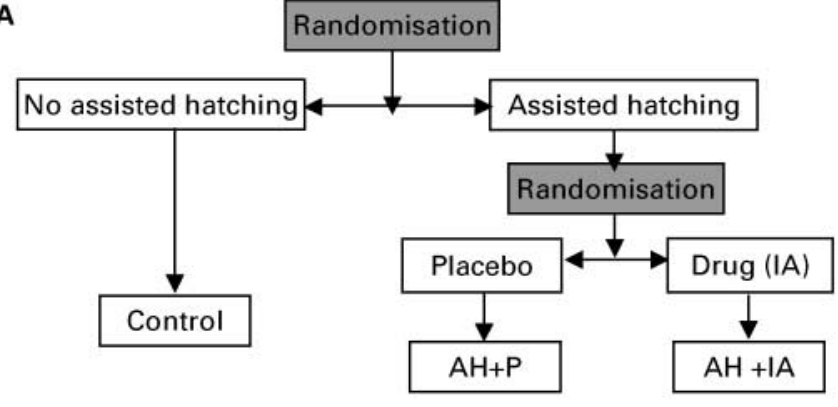

B
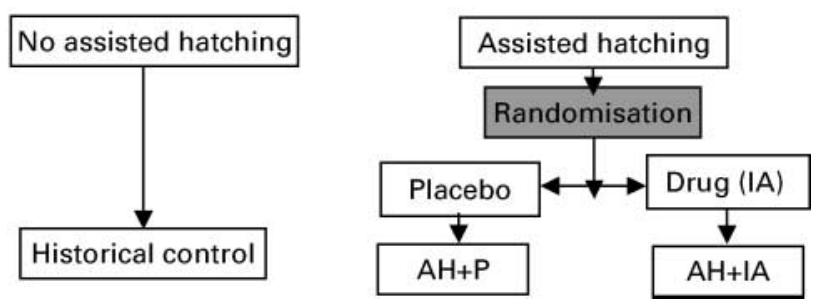

Figure 1. (A) Randomization scheme for Groups I and III. (B) Randomization scheme for Groups II and IV.

For ethical reasons all patients in Groups II and IV received the laser treatment. Therefore, historical control groups with patients treated during the same period of time and presenting the same inclusion criteria were built up retrospectively for these two groups.

In all four groups, the patients receiving laser-drilled embryos were further randomized in a double-blind trial, testing the role of an immunosuppressive/antibiotic treatment (IA) versus placebo (P) (Figure 1).

The patient data were collected in each centre on case report forms (CRFs). Regularly the CRFs were sent to the principal investigator of the study in Lausanne and data from each patient were reviewed and stored in a computerized database (FileMaker Pro, Filemaker Inc., USA) for further analysis.

The patient's anonymity was maintained, as patients were identified on CRFs by their initials and a code number. The coinvestigators kept a record of patient's name, address and code and only the CRFs bearing the patients' initials were sent to the principal investigator for analysis.

During the course of the treatment, the patient might have been discontinued for the following reasons: lack of ovulation, serious adverse effects, major protocol violation, discovery of ineligibility, non compliance, administrative reasons and pregnancy.

\section{AH using the $1.48 \mu \mathrm{m}$ diode laser}

The four centres involved in the study used the same laser device (Fertilase, Medical Technologies Montreux S.A., Clarens, Switzerland), which was installed on each co-investigator's inverted microscopes (Rink et al., 1994). The ZP was opened by drilling a tangential trench using a similar strategy. The culture dish was placed on the heated displacement stage of the microscope and a region of the $\mathrm{ZP}$, adjacent to a wide perivitelline space, was positioned at the location of the aiming spot. The ZP was fully dissected by 1-4 laser shots, in order to create a single breach presenting a width close to that of the ZP. Sizes of the drilled holes could be adapted by varying the irradiation time; typically a trench having a diameter of $20 \mu \mathrm{m}$ was produced by a $12-30 \mathrm{~ms}$ irradiation time (Rink et al., 1996). Because of the short irradiation time needed, no micromanipulators were necessary to stabilize the oocyte's position during the drilling procedure. When larger holes involving several 
laser shots were to be drilled, the embryos were placed in a $20-50 \mu \mathrm{l}$ drop of medium under mineral oil; undesirable displacements of the embryos due to thermal convection that are generally present inside larger culture dishes could thus be avoided. According to our previous experience on mouse embryos (Germond et al., 2000), the ZP has to be fully breached to be effective compared to local thinning, thus a hole diameter equal or superior to ZP thickness was recommended for ZP drilling in the study. However, it has been shown that thinning of the $\mathrm{ZP}$, but on a wider area (a quarter of the $\mathrm{ZP}$ ), was more effective than total breaching and local thinning (Mantoudis et al., 2001).

The safety and efficacy of the method have been previously established by determining the ability of mouse oocytes to fertilise in vitro and develop in vivo (Germond et al., 1995, 1996).

\section{Fresh embryo transfer}

Embryo transfers were performed on day 2 or 3 according to each centre's practice. AH was performed a few hours before embryo transfer. On that occasion, the embryos were examined and scored by recording the number and regularity of the blastomeres as well as the amount of extracellular fragments (Senn et al., 2000). The ZP thickness was measured on four opposite sides.

Progesterone was administered vaginally as a luteal phase supplementation and continued daily up to menstruation or for 10 weeks in pregnant patients.

\section{Frozen-thawed embryo transfers}

Zygotes or embryos were cryopreserved according to the same protocol in all centres, using propanediol as cryoprotectant, manual seeding at $-7^{\circ} \mathrm{C}$, and a slow freezing procedure lasting $\sim 90 \mathrm{~min}$ (from $22^{\circ} \mathrm{C}$ to $-150^{\circ} \mathrm{C}$ ). The devices used to perform the freezing were 'Air Liquide Minicool 40 PC' (Switzerland and Spain), 'Air Liquide LC 40' (France) and 'Labotect CTE 90' (Germany). Again, the thawing procedures were the same in all four centres. Zygotes cryopreserved at the pronucleate stage were thawed 1 day before embryo transfer in order to allow for the first cleavage to take place. Embryos cryopreserved at the cleavage stage were thawed according to their development stage at freezing and transferred accordingly. Transfers of frozen-thawed embryos were performed in natural or artificial cycles. AH and embryo transfers were performed under conditions similar to those used for fresh embryos.

\section{Drug treatment}

The additional medication consisted of methylprednisolone $16 \mathrm{mg}$ (Medrol ${ }^{\circledR}$, Pharmacia \& Upjohn AG, Switzerland) and doxycycline $200 \mathrm{mg}$ (Supracycline ${ }^{\circledR}$, Grünenthal Pharma AG, Switzerland) which were given 2 days prior and up to 5 days after ET. According to a randomization list, the pharmacy at the CHUV prepared for each patient the set of drugs necessary for completing one treatment cycle. All drugs were reconditioned in packages, allowing full blinding both for the medical staff and the patient.

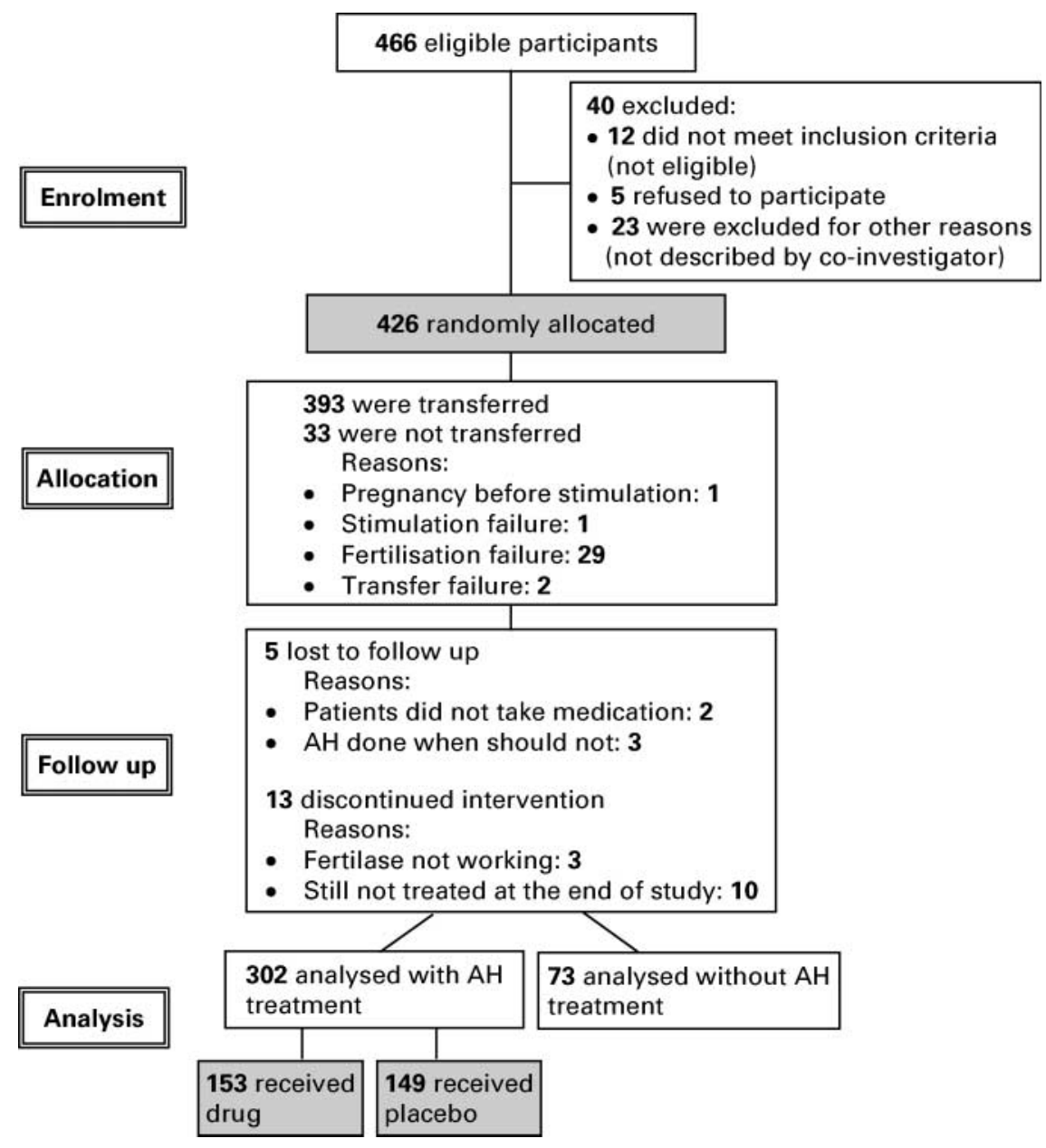

Figure 2. Flow diagram of the multicentre trial comparing the effect of assisted hatching on four different patient populations. 


\section{Randomization}

The number of cases $(n)$ necessary to achieve statistical significance $(P<0.05)$ when a $20 \%$ increase in pregnancy rate after $\mathrm{AH}$ is expected was estimated as follows:

Group I: Control $(n=70) ; \mathrm{AH}+\mathrm{P}(n=70) ; \mathrm{AH}+\mathrm{IA}(n=70)$

Group II: $\mathrm{AH}+\mathrm{P}(n=70) ; \mathrm{AH}+\mathrm{IA}(n=70)$

Group III: Control $(n=50) ; \mathrm{AH}+\mathrm{P}(n=50) ; \mathrm{AH}+\mathrm{IA}(n=50)$

Group IV: $\mathrm{AH}+\mathrm{P}(n=50) ; \mathrm{AH}+\mathrm{IA}(n=50)$

Groups I and III were randomized both for $\mathrm{AH}$ and medication according to the scheme described in Figure 1A. Groups II and IV were randomized only for IA because all patients received $\mathrm{AH}$, as shown in Figure 1B. Computer-generated randomization lists were drawn up separately for $\mathrm{AH}$ and drug assignments for each treatment group. The information relative to the $\mathrm{AH}$ procedure was sent to the centres by lists of 10 case numbers in sealed envelopes. The local medical supervisors in charge of the study allocated the next available case number on the list to each admitted couple and distributed the box of tablets bearing the same case number. Case numbers and patient initials were recorded by the study supervisor on the CRF and were used as means to unequivocally identify a patient.

According to the CONSORT (Consolidated Standards of Reporting Trials) statement for randomized studies (Altman et al., 2001), a flow diagram of the progress through the phases of this randomized trial is provided in Figure 2. Out of the 600 patients initially expected, 466 could be considered eligible by the co-investigators and 426 were randomly allocated between January 1997 and December 1999. The study was prematurely interrupted due to recruitment difficulties.

\section{Blinding}

Participants and medical supervisors were blinded in respect to both the AH procedure and the IA treatment. Patients were only aware of the group they had been allocated to. Laboratory staff was necessarily aware of the $\mathrm{AH}$ assignment according to the randomization list, but was blind to the IA/placebo treatment. The randomization list for the drug treatment was kept by the pharmacy department of the CHUV and unveiled at the end of the study.

\section{Outcome measures}

The primary outcome measure was the occurrence of a clinical pregnancy diagnosed by elevated serum hCG levels and the presence of at least one fetal sac exhibiting cardiac activity. The clinical pregnancy (CPR) and implantation (IR) rates were calculated for each subgroup of patients (Control, $\mathrm{AH}+\mathrm{P}$ and $\mathrm{AH}+\mathrm{IA}$ ). Assessment of efficacy and safety were also based on the following secondary outcome measures: live births, abortions, multiple pregnancies, biochemical pregnancies (miscarriage without evidence of a fetal sac on ultrasound and hCG levels remaining $<1000 \mathrm{UI} / 1$ and reaching undetectable values 3 weeks after transfer).

\section{Statistical analysis}

Chi-square and Student's $t$-test were calculated wherever appropriate with the StatView Student statistical package. Differences were considered as significant when $P$ reached values $<0.05$.

\section{Results}

Table I summarizes some relevant clinical aspects of the treated patients in the four groups. Patients in Groups I and II were comparable in terms of age, body mass index (BMI),
Table I. Clinical data regarding the four groups of treated patients

\begin{tabular}{|c|c|c|c|c|}
\hline & \multicolumn{4}{|l|}{ Groups } \\
\hline & I & II & III & IV \\
\hline $\begin{array}{l}\text { Number of } \\
\text { expected patients }\end{array}$ & 210 & 140 & 150 & 100 \\
\hline $\begin{array}{l}\text { Number of treated } \\
\text { patients }\end{array}$ & 171 & 75 & 66 & 65 \\
\hline Age $($ mean $\pm \mathrm{SD})$ & $32.4 \pm 3.9$ & $32.3 \pm 4.0$ & $39.3 \pm 2.8$ & $35.1 \pm 4.1$ \\
\hline $\mathrm{BMI}($ mean $\pm \mathrm{SD})$ & $22.5 \pm 3.7$ & $21.7 \pm 3.1$ & $23.5 \pm 4.1$ & $22.7 \pm 3.8$ \\
\hline Smokers & $39 \%$ & $34 \%$ & $36 \%$ & $23 \%$ \\
\hline Primary infertility & $73 \%$ & $68 \%$ & $63 \%$ & $72 \%$ \\
\hline \multicolumn{5}{|c|}{ IVF indications, $n(\%)$} \\
\hline Female & $52(30.4)$ & $31(41.3)$ & $26(39.4)$ & $14(21.5)$ \\
\hline Male & $97(56.7)$ & $30(40.0)$ & $18(27.3)$ & $34(52.3)$ \\
\hline Mixed & $11(6.4)$ & $9(12.0)$ & $5(7.6)$ & $4(6.2)$ \\
\hline Idiopathic & $11(6.4)$ & $5(6.7)$ & $17(25.8)$ & $13(20.0)$ \\
\hline
\end{tabular}

occurrence of primary infertility and smoking habit, as well as IVF indications. Patients in Groups III and IV were significantly older $(P<0.05)$ and presented a higher incidence of idiopathic causes of infertility $(P<0.05)$.

The clinical outcome of the transfers in the four groups of patients treated is presented in Table II. In Group I (Table IIA), significantly lower pregnancy (1.6\% versus $15.1 \% ; P=0.0195)$ and implantation $(1.6 \%$ versus $10.0 \%$; $P=0.0106)$ rates were obtained in the subgroup of patient receiving laser-drilled embryos and a placebo $(\mathrm{AH}+\mathrm{P})$ compared to the control. These rates were restored to those of the control (10.7\% versus $15.1 \%$ and $6.0 \%$ versus $10.0 \%$ ) when AH was accompanied by corticosteroid/antibiotic administration (AH + IA). All other parameters, such as age, number of transferred embryos and ZP thickness did not differ significantly among the three subgroups (control, $\mathrm{AH}+\mathrm{P}$ and $\mathrm{AH}+\mathrm{IA})$. The mean hole diameters of the $\mathrm{AH}$ embryos were close to the mean ZP thickness and statistically similar in both subgroups $\mathrm{AH}+\mathrm{P}$ and $\mathrm{AH}+\mathrm{IA}$.

In Group II (Table II.B.), both clinical pregnancy $(7.9 \%$ versus $10.8 \%)$ and implantation rates $(5.1 \%$ versus $4.8 \%)$ did not differ among the patients in $\mathrm{AH}+\mathrm{IA}$ and $\mathrm{AH}+\mathrm{P}$. These rates were furthermore quite similar to the historical control group. As for Group I, all other parameters recorded did not differ significantly among the three subgroups of patients.

Among the poor prognosis patients (Group III, Table IIC), slightly lower but not statistically different rates in the clinical pregnancies and implantation were observed in the $\mathrm{AH}+\mathrm{P}$ subgroup compared to the control (13.6\% versus $23.8 \%$ and $6.4 \%$ versus $9.1 \%$ ) and to the $\mathrm{AH}+\mathrm{IA}$ subgroup (13.6\% versus $21.7 \%$ and $6.4 \%$ versus $9.1 \%$ ). The $\mathrm{AH}+\mathrm{IA}$ subgroup yielded rates similar to the control. However, the 'take home baby rate' was increased, but not significantly, after $\mathrm{AH}+\mathrm{IA}$ due to only one abortion in this subgroup compared to four in the control one.

Finally, in Group IV (Table IID), both clinical pregnancy $(16.7 \%$ and $14.3 \%$ versus $11.4 \%$ ) and implantation $(7.5 \%$ and $6.3 \%$ versus $5.2 \%$ ) rates were slightly higher after $\mathrm{AH}+\mathrm{IA}$ and $\mathrm{AH}+\mathrm{P}$ compared to the historical control group. As for the other groups, all other parameters recorded 


\begin{tabular}{|c|c|c|c|}
\hline A: Group I & Control & $\mathrm{AH}+\mathrm{P}$ & $\mathrm{AH}+\mathrm{IA}$ \\
\hline Number of patients & 53 & 62 & 56 \\
\hline Mean age (years) & $32.6 \pm 3.8$ & $32.8 \pm 4.2$ & $31.7 \pm 3.7$ \\
\hline Total embryos transferred & 110 & 127 & 116 \\
\hline $\mathrm{ZP}$ thickness $(\mu \mathrm{m})$ & $17.9 \pm 3.3$ & $17.3 \pm 3.1$ & $17.0 \pm 2.4$ \\
\hline Hole diameter $(\mu \mathrm{m})$ & & $17.9 \pm 6.1$ & $18.0 \pm 7.1$ \\
\hline Mean embryos transferred & $2.1 \pm 0.7$ & $2.0 \pm 0.7$ & $2.1 \pm 0.7$ \\
\hline Biochemical pregnancies $n(\%)$ & $3(5.7)$ & $1(1.6)$ & $2(3.6)$ \\
\hline Clinical pregnancies $n(\%)$ & $8(15.1)^{\mathrm{a}}$ & $1(1.6)^{\mathrm{a}}$ & $6(10.7)$ \\
\hline Abortions $n$ & 1 & 0 & 2 \\
\hline Deliveries $n(\%)$ & $7(13.2)$ & $1(1.6)$ & $4(7.1)$ \\
\hline Multiple pregnancies $n$ & 3 & 1 & 1 \\
\hline Number of sacs $n$ & 11 & 2 & 7 \\
\hline Implantation rate $n(\%)$ & $10.0^{\mathrm{b}}$ & $1.6^{\mathrm{b}}$ & 6.0 \\
\hline B: Group II & Historical control & $\mathrm{AH}+\mathrm{P}$ & $\mathrm{AH}+\mathrm{IA}$ \\
\hline Number of patients & 45 & 37 & 38 \\
\hline Mean age (years) & $31.3 \pm 3.3$ & $33.3 \pm 4.2$ & $31.4 \pm 3.5$ \\
\hline Total embryos transferred & 88 & 83 & 79 \\
\hline $\mathrm{ZP}$ thickness $(\mu \mathrm{m})$ & & $17.2 \pm 4.6$ & $17.4 \pm 4.2$ \\
\hline Hole diameter $(\mu \mathrm{m})$ & & $18.0 \pm 11.4$ & $18.4 \pm 11.6$ \\
\hline Mean embryos transferred & $2.0 \pm 0.7$ & $2.2 \pm 0.6$ & $2.1 \pm 0.7$ \\
\hline Biochemical pregnancies $n(\%)$ & $0(0.0)$ & $0(0.0)$ & $0(0.0)$ \\
\hline Clinical pregnancies $n(\%)$ & $4(8.9)$ & $4(10.8)$ & $3(7.9)$ \\
\hline Abortions $n$ & 0 & 0 & 0 \\
\hline Deliveries $n(\%)$ & $4(8.9)$ & $4(10.8)$ & $3(7.9)$ \\
\hline Multiple pregnancies $n$ & 1 & 0 & 1 \\
\hline Number of sacs $n$ & 5 & 4 & 4 \\
\hline Implantation rate (\%) & 5.7 & 4.8 & 5.1 \\
\hline C: Group III & Control & $\mathrm{AH}+\mathrm{P}$ & $\mathrm{AH}+\mathrm{IA}$ \\
\hline Number of patients & 21 & 22 & 23 \\
\hline Mean age (years) & $38.3 \pm 3.4$ & $39.3 \pm 2.9$ & $40.1 \pm 2.0$ \\
\hline Total embryos transferred & 55 & 47 & 55 \\
\hline ZP thickness $(\mu \mathrm{m})$ & $16.9 \pm 3.6$ & $16.4 \pm 3.6$ & $15.8 \pm 4.2$ \\
\hline Hole diameter $(\mu \mathrm{m})$ & & $16.8 \pm 8.5$ & $16.5 \pm 11.1$ \\
\hline Mean embryos transferred $n$ & $2.6 \pm 0.5$ & $2.1 \pm 0.9$ & $2.4 \pm 0.7$ \\
\hline Biochemical pregnancies $n(\%)$ & $4(19.0)$ & $0(0.0)$ & $1(4.3)$ \\
\hline Clinical pregnancies $n(\%)$ & $5(23.8)$ & $3(13.6)$ & $5(21.7)$ \\
\hline Abortions $n$ & 4 & 1 & 1 \\
\hline Deliveries $n(\%)$ & $1(4.8)$ & $2(9.1)$ & $4(17.4)$ \\
\hline Multiple pregnancies $n$ & 0 & 0 & 0 \\
\hline Number of sacs $n$ & 5 & 3 & 5 \\
\hline Implantation rate $n(\%)$ & 9.1 & 6.4 & 9.1 \\
\hline D: Group IV & Historical control & $\mathrm{AH}+\mathrm{P}$ & $\mathrm{AH}+\mathrm{IA}$ \\
\hline Number of patients & 44 & 30 & 35 \\
\hline Mean age (years) & $34.4 \pm 4.1$ & $34.5 \pm 3.8$ & $35.6 \pm 4.2$ \\
\hline Total embryos transferred & 115 & 80 & 96 \\
\hline $\mathrm{ZP}$ thickness $(\mu \mathrm{m})$ & & $16.9 \pm 3.1$ & $16.7 \pm 4.5$ \\
\hline Hole diameter $(\mu \mathrm{m})$ & & $18.0 \pm 3.2$ & $18.0 \pm 8.0$ \\
\hline Mean embryos transferred & $2.6 \pm 0.7$ & $2.7 \pm 0.5$ & $2.7 \pm 0.4$ \\
\hline Biochemical pregnancies $n(\%)$ & $1(2.3)$ & $2(6.7)$ & $2(5.7)$ \\
\hline Clinical pregnancies $n(\%)$ & $5(11.4)$ & $5(16.7)$ & $5(14.3)$ \\
\hline Abortions $n$ & 2 & 1 & 1 \\
\hline Deliveries $n(\%)$ & $3(6.8)$ & $4(13.3)$ & $4(11.4)$ \\
\hline Multiple pregnancies $n$ & 1 & 1 & 0 \\
\hline Number of sacs $n$ & 6 & 6 & 6 \\
\hline Implantation rate (\%) & 5.2 & 7.5 & 6.3 \\
\hline
\end{tabular}

Control: intact embryos; $\mathrm{AH}+\mathrm{P}$ : laser drilled embryos with placebo; $\mathrm{AH}+\mathrm{IA}$ : laser drilled embryos with immunosuppressive/antibiotic treatment; Historical control: intact embryos.

${ }^{\mathrm{a}} P=0.0195$.

${ }^{\mathrm{b}} P=0.0106$.

did not differ significantly among the three subgroups of patients.

In order to reduce the confounding influence of the embryo quality, data from Groups I and II were pooled and the pool of transfers meeting the following criteria was extracted: (i) cryopreservation was performed at the pronucleate stage; (ii) transfers, performed on day 2 or 3 contained at least one good quality embryo (day 2: four blastomeres with no or 
Table III. Clinical outcome of selected transfers containing at least one embryo of good quality (4I-4II on day 2 or 8I-8II on day 3 ) after cryopreservation at the pronucleate stage, Groups I and II combined

\begin{tabular}{llll}
\hline Groups I and II & Control & $\mathrm{AH}+\mathrm{P}$ & $\mathrm{AH}+\mathrm{IA}$ \\
\hline Number of patients & 46 & 29 & 34 \\
Mean age (years) & $31.0 \pm 3.8$ & $33.4 \pm 3.9$ & $31.2 \pm 3.2$ \\
Total embryos transferred & 96 & 64 & 67 \\
ZP thickness $(\mu \mathrm{m})$ & & $15.9 \pm 3.2$ & $16.9 \pm 4.5$ \\
Mean embryos transferred & $2.1 \pm 0.6$ & $2.2 \pm 0.6$ & $2.0 \pm 0.6$ \\
Biochemical pregnancies $n(\%)$ & $2(4.3)$ & $2(6.9)$ & $1(2.9)$ \\
Clinical pregnancies $n(\%)$ & $9(19.6)$ & $2(6.9)$ & $5(14.7)$ \\
Abortions $n$ & $1(11.1)$ & $1(50.0)$ & $1(20.0)$ \\
Deliveries $n(\%)$ & $8(17.4)$ & $1(3.4)$ & $4(11.8)$ \\
Multiple pregnancies $n$ & $3(33.3)$ & $0(0.0)$ & $0(0.0)$ \\
Number of sacs $n$ & 12 & 3 & 5 \\
Implantation rate $(\%)$ & 12.5 & 4.7 & 7.5 \\
\hline
\end{tabular}

Control: intact embryos; $\mathrm{AH}+\mathrm{P}$ : laser drilled embryos with placebo; $\mathrm{AH}+\mathrm{IA}$ : laser drilled embryos with immunosuppressive/antibiotic treatment.
Table IV. Clinical outcome of all transfers, fresh and cryopreserved combined, classified according to the mean ZP thickness of the transferred embryos and to the type of medication.

\begin{tabular}{llll}
\hline All groups & \multicolumn{2}{l}{ Mean ZP thickness } & \multirow{2}{*}{$P$} \\
\cline { 2 - 3 } & $<17 \mu \mathrm{m}$ & $17 \mu \mathrm{m}$ & \\
\hline Cycles AH + P $n$ & 71 & 78 & $\mathrm{NS}$ \\
Clinical pregnancies & $6 / 71(8.5 \%)$ & $6 / 78(7.7 \%)$ & $\mathrm{NS}$ \\
Implantation rates & $7 / 158(4.4 \%)$ & $7 / 174(4.0 \%)$ & \\
\hline Cycles AH + IA $n$ & 78 & 73 & $\mathrm{NS}$ \\
Clinical pregnancies & $10 / 78(12.8 \%)$ & $9 / 73(12.3 \%)$ & $\mathrm{NS}$ \\
Implantation rates & $11 / 180(6.1 \%)$ & $11 / 163(6.7 \%)$ & \\
\hline
\end{tabular}

$\mathrm{AH}+\mathrm{P}$ : laser drilled embryos with placebo; AH + IA: laser drilled embryos with immunosuppressive/antibiotic treatment. Diode laser for assisted hatching and immunosuppression in a randomized study.

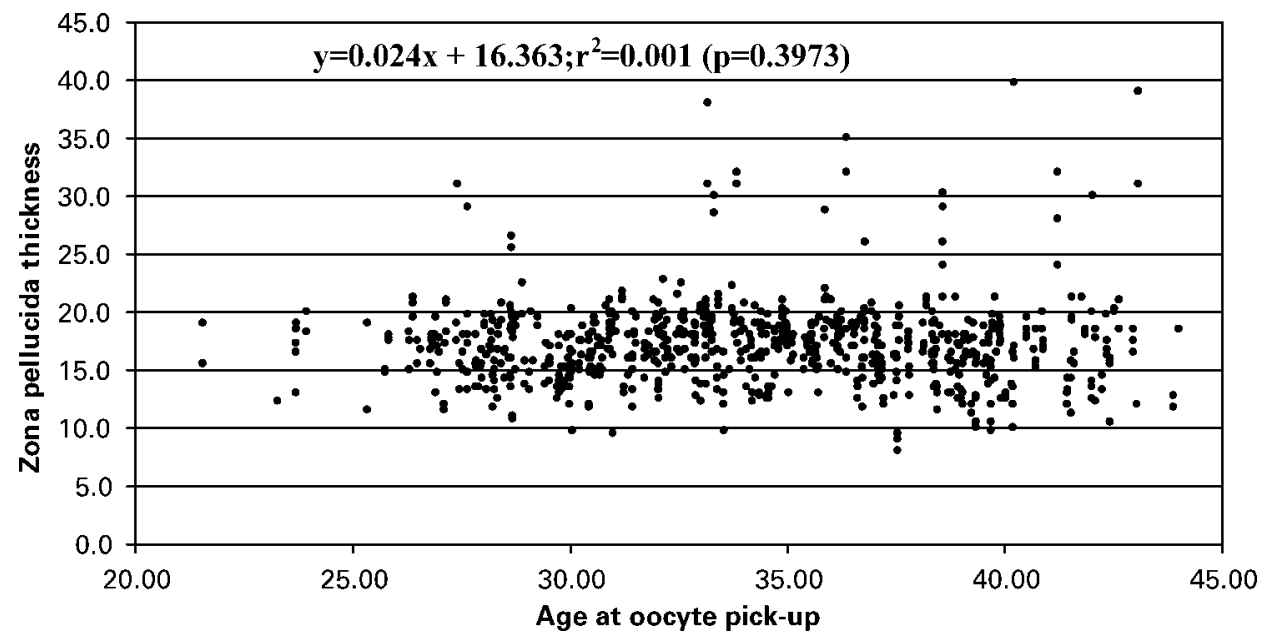

Figure 3. Scattergram of the ZP thickness in relation to age of the patient at OPU. $n=363$.

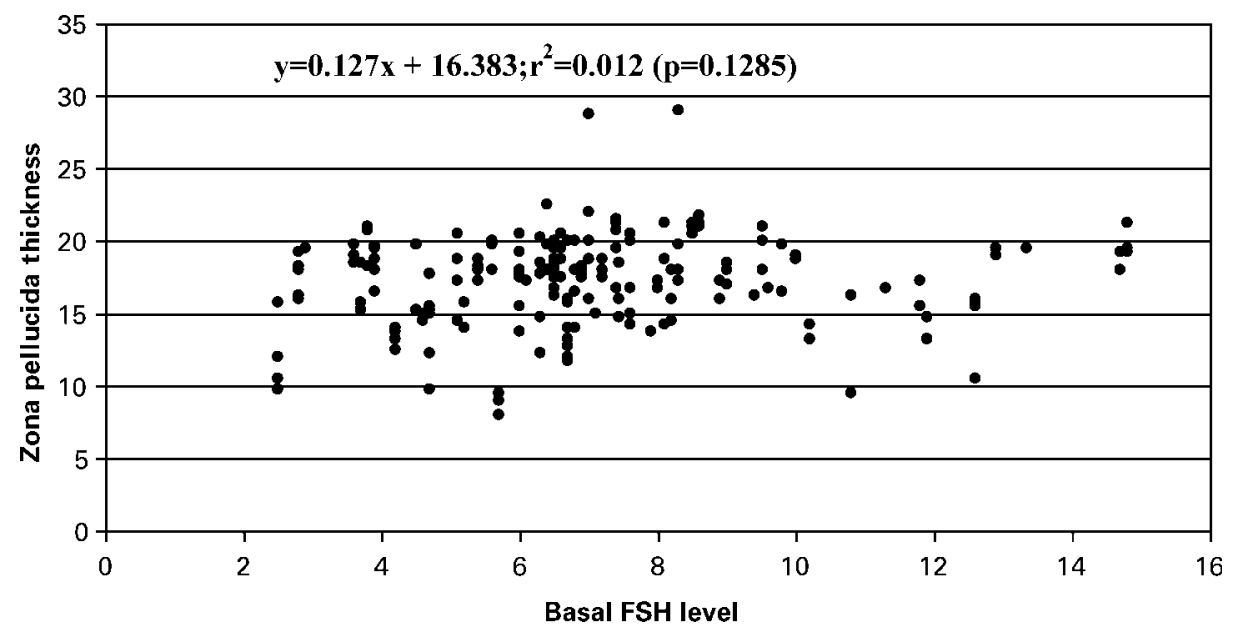

Figure 4. Scattergram of the ZP thickness (in $\mu \mathrm{m}$ ) in relation to basal FSH (UI/l) of the patient. Restricted to those patients for which the value of the FSH level was known. $n=192$. 


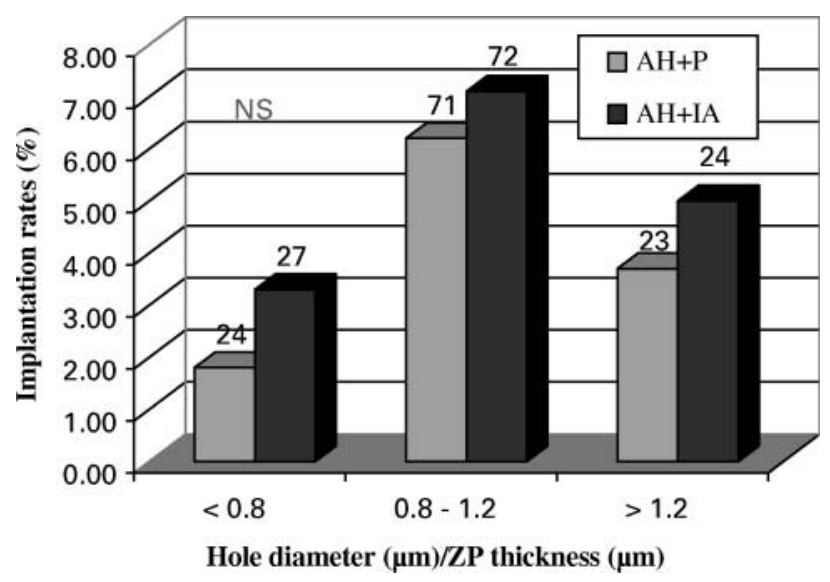

Figure 5. Implantation rates after transfers of embryos receiving assisted hatching with different hole sizes in patients treated (IA) or not (Placebo) with the immunosuppressive/antibiotic treatment. $\mathrm{NS}=$ not significantly different.

little fragments; day 3: eight blastomeres with no or little fragments). Clinical outcome of the embryo transfers are presented in Table III. No significant difference was observed between the subgroups in terms of clinical pregnancy and implantation rates, although $\mathrm{AH}$ in the absence of immunosuppressive treatment led to a lower pregnancy rate $(6.9 \%$ versus $19.6 \%$ ).

The relationship between $\mathrm{ZP}$ thickness and age of the patients at OPU has been studied by combining the values measured in all groups. The scattergram presented in Figure 3 indicates that the $\mathrm{ZP}$ thickness was not related to the age of the patients in the study population. A similar observation can be made when the ZP thickness is expressed relatively to the basal FSH level (Figure 4).

In order to test whether the ZP thickness was related to the clinical outcome after $\mathrm{AH}$, pregnancy and implantation rates have then been calculated when the mean $\mathrm{ZP}$ was $<17 \mu \mathrm{m}$ or above. Results, presented in Table IV, indicate that both rates were similar when $\mathrm{AH}$ was performed on thin or thick $\mathrm{ZP}$, independently of the IA treatment.

The implantation rates of the embryos having been treated with $\mathrm{AH}$ in all the groups were studied in relation to the size of the hole performed for $\mathrm{AH}$ (Figure 5). Although the rates showed no statistical difference, the embryos with the diameter of the hole the nearest to the ZP thickness (Hole diameter/ZP thickness close to 1) were implanting with a higher rate than the ones with a smaller or a bigger opening.

The numbers of biochemical pregnancies and abortions were too small to draw any conclusions and the values obtained were comparable among the subgroups and between the groups (Table IIA-D).

\section{Discussion}

The data obtained in this prospective randomized study indicate that $\mathrm{AH}$ does not lead to higher implantation rates in older patients (Group III) and in patients initiating a cryo cycle (Groups I and II). In two groups, I and III, AH treatment appears to have a negative effect when not supported by IA therapy. When $\mathrm{AH}$ is performed on fresh embryos after repeated implantation failures (Group IV), the implantation and clinical pregnancy rates are both improved although not significantly. In the latter case, the IA treatment does not add any positive effect on the rates.

Recruitment of the patients for this study appeared much more difficult than initially anticipated. The study had thus to be ended before the expected number of patients $(n=600)$ was reached. Despite the premature ending of the study, there was no negative impact on the statistical significance of the data.

The study was elaborated in order to confirm results obtained on smaller groups of patients in two of the European centres. One study performed in the IVF centre of Lausanne on a group of patients similar to Group II had shown that patients receiving a transfer of good quality frozen-thawed embryos gained from the ZP microdissection treatment compared to control (Germond et al., 2000). When the embryo quality was high (CES 20), AH increased IR from $3.6 \%$ to $14.7 \%$ and CPR from $10.5 \%$ to $29.6 \%$. Sixteen deliveries were obtained with the birth of 22 healthy children in the $\mathrm{AH}$ group, compared to four deliveries and four children in the control group (Germond et al., 2000). However, when the four IVF centres tried to confirm these findings, the results were very different and no improvement was observed after $\mathrm{AH}$ even in good score embryo transfers. The other prospective study performed in the IVF centre of Bonn, on two groups of patients with advanced maternal age, demonstrated that $\mathrm{AH}$ increased both implantation and clinical pregnancy rates (Montag and van der Ven, 1999). There again, these findings were not confirmed in the multicentre study where AH did not improve the clinical outcome of older patients. However, it should be noted that in the study by Montag and van der Ven (1999), although patients were selected according to maternal age, they had also failed treatment cycles in their past, so that it is not possible to compare these results directly to those of Group III in the multicentre study.

In general, the disparity between the positive results of these two small (small number of patients and/or study performed in a single centre) prospective but not randomized studies and the null ones obtained in the multicentre randomized study with similar groups of patients shows that the results obtained within a group of patients, in a particular laboratory and with a specific technique, cannot be generalized. In the same way, several clinical studies have been performed for the last decade in different IVF centres and demonstrated that $\mathrm{AH}$ could either have a positive or a null effect on IR and CPR rates when the same groups of patients were selected (Germond et al., 1999). Similarly to the present work, a multicentre study (two IVF centres) reported that $\mathrm{AH}$ was unable to benefit patients selected for advanced age, zona thickness and previous multiple failures in both fresh and frozen-thawed embryos (Edirisinghe et al., 1999). However, two meta-analyses by Edi-Osagie et al. (2003) and Sallam et al. (2003) show a positive trend of AH for patients similar to those of Group IV with repeated implantation failures. 
It is obvious in this study that patients receiving a transfer of ZP microdissected embryos needed a concomitant administration of an immunosuppressive/antibiotic therapy. The treatment of patients with corticosteroids may prevent some undefined antibodies or surveillance cells from gaining access to and destroying the embryos. This may be especially important after zona drilling (Cohen et al., 1990) or blastocyst natural hatching when the embryo is left exposed to uterine lymphocytes and immune cells.

Secondary objectives were to establish the roles of the morphological characteristics of the transferred embryos, the ZP thickness and the opening diameter on the implantation rates and to initiate a follow-up of the pregnancies up to delivery. It is possible that the efficacy of AH depends less on the clinical variables of the patients than on the morphological characteristics of oocytes and embryos (Montag et al., 2000; Ebner et al., 2002). AH is not going to ameliorate the poor quality of an embryo or improve its development up to the blastocyst stage. A better selection of the embryos already guarantees higher implantation and clinical pregnancy rates. However, in the present work, no embryo selection was performed in Lausanne and Bonn due to legal restrictions and all the embryos, kept fresh in culture or thawed, were transferred. This might explain why the implantation rates observed in this study were much lower than those obtained when embryo selection is common practice.

The safety of the laser had already been proved in animal studies first (Germond et al., 1995, 1996) and then in humans (Germond et al., 2000). The results of this study show that the $\mathrm{AH}$ treatment does not increase the abortion rate or the number of biochemical pregnancies, thus confirming the safety of the technique. A children follow-up study (results not published) has been performed in the Lausanne IVF centre on 51 deliveries and 62 babies born after $\mathrm{AH}$ and looking at prenatal karyotypes, sex ratio, birth parameters, major and minor congenital malformations and physical examinations of the children after birth and 4-6 weeks later. No higher risks for incidence of congenital malformation and chromosomal aberration were found in that group of children compared to control. A similar study was performed in Budapest (Kanyo and Konc, 2003) with 96 deliveries and 134 babies born, which conclude the same results.

The number of multiple pregnancies was not higher in the $\mathrm{AH}$ treated patients than in the control patients. One fear is that AH could increase the incidence of monozygotic twinning (MZT) due to a partial hatching of the conceptus through the hole made in ZP causing a herniation and a division into two embryos. Several studies have been published which tend to demonstrate that the frequency of MZT is not different after the transfers of 'zona manipulated' embryos compared to 'zona intact' ones (Sills et al., 2000) and that if the rate of MZT is increased after assisted reproduction, it is irrespective of the treatment modality or the type of micromanipulation (Schachter et al., 2001). Only a few trials reported an increase in MZT after AH (Hershlag et al., 1999; Yakin et al., 2003) and most of them are case reports (Schieve et al., 2000; Sheen et al., 2001). Three other studies
(Behr et al., 2000; da Costa et al., 2001; Milki et al., 2003) showed that transfers at the blastocyst stage were the main reason for an increase in the frequency of MZT.

In conclusion, this study did not succeed in clearly identifying one or more groups of patients in need of $\mathrm{AH}$ treatment, neither in designating the embryos with specific morphometric characteristics that predestined them to hatching failure. Patients with repeated unexplained IVF failure constitute the main target group for ZP microdissection, but ideally the patients or the embryos requiring $\mathrm{AH}$ should be identified before the unsuccessful attempts. Clinically, the indications for $\mathrm{AH}$ still have to be weighed according to a series of confounding variables, such as the number of unsuccessful embryo transfers, the patient's age, the morphological characteristics of the embryo and the $\mathrm{ZP}$, keeping in mind that no harm can be done to the embryo by practising $\mathrm{AH}$, that the incidence of monozygotic twinning is not increased and that the implantation is not impaired as long as an immunosuppressive/antibiotic treatment is prescribed.

\section{Acknowledgements}

We are grateful to K. Rink and G. Delacrétaz from the Applied Optics Institute, EPFL, CH-1015 Ecublens, Switzerland, who assisted the four laboratories with their technical skills on the diode laser. We thank A. Pannatier from the Pharmacy, CHUV, CH-1011 Lausanne, Switzerland, who was in charge of the randomization lists and the preparation of the drugs. We acknowledge the great help of D. Goy with the statistical analysis and F. Urner with reading and commenting on the manuscript. Finally, we would like to thank the medical doctors who recruited the patients and the laboratory technicians who performed the laser assisted hatching, from all four IVF centres.

\section{References}

Abdalla HI, Brooks AA, Johnson MR, Kirkland A, Thomas A and Studd JW (1994) Endometrial thickness: a predictor of implantation in ovum recipients? Hum Reprod 9,363-365.

Altman DG, Schulz KF, Moher D, Egger M, Davidoff F, Elbourne D, Gotzsche PC and Lang T (2001) The revised CONSORT statement for reporting randomized trials: explanation and elaboration. Ann Intern Med $134,663-694$.

Balaban B, Urman B, Alatas C, Mercan R, Mumcu A and Isiklar A (2002) A comparison of four different techniques of assisted hatching. Hum Reprod $17,1239-1243$.

Baruffi RL, Contart P, Mauri AL, Petersen C, Felipe V, Garbellini E and Franco JG (2002) A uterine ultrasonographic scoring system as a method for the prognosis of embryo implantation. J Assist Reprod Genet 19, 99-102.

Behr B, Fisch JD, Racowsky C, Miller K, Pool TB and Milki AA (2000) Blastocyst-ET and monozygotic twinning. J Assist Reprod Genet 17, $349-351$

Bongso A, Ng SC, Fong CY and Ratnam S (1991) Cocultures: a new lead in embryo quality improvement for assisted reproduction [see comments]. Fertil Steril 56,179-191.

Chao KH, Chen SU, Chen HF, Wu MY, Yang YS and Ho HN (1997) Assisted hatching increases the implantation and pregnancy rate of in vitro fertilization (IVF)-embryo transfer (ET), but not that of IVF-tubal ET in patients with repeated IVF failures. Fertil Steril 67,904-908.

Cohen J (1991) Assisted hatching of human embryos. J In Vitro Fert Embryo Transf 8,179-190.

Cohen J (1993) Assisted hatching: indications and techniques. Acta Eur Fertil 24,215-219.

Cohen J, Alikani M, Reing AM, Ferrara TA, Trowbridge $\mathrm{J}$ and Tucker M (1992) Selective assisted hatching of human embryos. Ann Acad Med Singapore 21,565-570. 
Cohen J, Malter H, Elsner C, Kort H, Massey J and Mayer MP (1990) Immunosuppression supports implantation of zona pellucida dissected human embryos. Fertil Steril 53,662-665.

da Costa AA, Abdelmassih S, de Oliveira FG, Abdelmassih V, Abdelmassih R, Nagy ZP and Balmaceda JP (2001) Monozygotic twins and transfer at the blastocyst stage after ICSI. Hum Reprod 16,333-336.

De Vos A and Van Steirteghem A (2000) Zona hardening, zona drilling and assisted hatching: new achievements in assisted reproduction. Cells Tissues Organs 166,220-227.

Ebner T, Moser M, Yaman C, Sommergruber M, Hartl J, Jesacher K and Tews G (2002) Prospective hatching of embryos developed from oocytes exhibiting difficult oolemma penetration during ICSI. Hum Reprod 17, 1317-1320.

Edi-Osagie E, Hooper L, McGinlay P and Seif M (2003) Effect(s) of assisted hatching on assisted conception (IVF \& ICSI). Cochrane Database Syst Rev 4, CD001894.

Edirisinghe WR, Ahnonkitpanit V, Promviengchai S, Suwajanakorn S, Pruksananonda K, Chinpilas V and Virutamasen P (1999) A study failing to determine significant benefits from assisted hatching: patients selected for advanced age, zonal thickness of embryos, and previous failed attempts. J Assist Reprod Genet 16,294-301.

Germond M, Nocera D, Senn A, Rink K, Delacretaz G and Fakan S (1995) Microdissection of mouse and human zona pellucida using a 1.48-microns diode laser beam: efficacy and safety of the procedure. Fertil Steril 64, 604-611.

Germond M, Nocera D, Senn A, Rink K, Delacretaz G, Pedrazzini T and Hornung JP (1996) Improved fertilization and implantation rates after nontouch zona pellucida microdrilling of mouse oocytes with a 1.48 microm diode laser beam. Hum Reprod 11,1043-1048.

Germond M, Primi MP, Senn A, Rink K and Delacrétaz G (1999) The use of laser for micromanipulation. In Shoham Z, Howles CM, and Jacobs S (eds) Female Infertility Therapy Current Practice. Martin Dunitz, London, UK, pp. 221-232.

Germond M, Primi MP, Senn A, Rink K, Descloux L and Delacrétaz G (2000) Diode laser for assisted hatching. In Wyss P, Tadir Y, Tromberg BJ, and Haller U (eds) Photomedicine in Gynecology and Reproduction. Karger, Basel,CH, pp. 352-365.

Gianaroli L, Magli MC, Ferraretti AP and Munne S (1999) Preimplantation diagnosis for aneuploidies in patients undergoing in vitro fertilization with a poor prognosis: identification of the categories for which it should be proposed. Fertil Steril 72,837-844.

Hershlag A, Paine T, Cooper GW, Scholl GM, Rawlinson K and Kvapil G (1999) Monozygotic twinning associated with mechanical assisted hatching. Fertil Steril 71,144-146.

Isik AZ, Vicdan K, Kaba A and Dagli G (2000) Comparison of zona manipulated and zona intact blastocyst transfers: a prospective randomized trial. J Assist Reprod Genet 17,135-139.

Kanyo K and Konc J (2003) A follow-up study of children born after diode laser assisted hatching. Eur J Obstet Gynecol Reprod Biol 110,176-180.

Lee KA, Koo JJ, Yoon TK, Do BR, Ko JJ and Cha KY (1994) Immunosuppression by corticosteroid has no effect on the pregnancy rate in routine in-vitro fertilization/embryo transfer patients. Hum Reprod 9,1832-1835.

Mantoudis E, Podsiadly BT, Gorgy A, Venkat G and Craft IL (2001) A comparison between quarter, partial and total laser assisted hatching in selected infertility patients. Hum Reprod 16,2182-2186.
Milki AA, Jun SH, Hinckley MD, Behr B, Giudice LC and Westphal LM (2003) Incidence of monozygotic twinning with blastocyst transfer compared to cleavage-stage transfer. Fertil Steril 79,503-506.

Montag M, Koll B and van der Ven H (2000) Use of a laser to evaluate zona pellucida hardness at different stages of mouse embryonic development in vitro and in vivo. J Assist Reprod Genet 17,178-181.

Montag M and van der Ven H (1999) Laser-assisted hatching in assisted reproduction. Croat Med J 40,398-403.

Nakayama T, Fujiwara H, Yamada S, Tastumi K, Honda T and Fujii S (1999) Clinical application of a new assisted hatching method using a piezo-micromanipulator for morphologically low-quality embryos in poorprognosis infertile patients. Fertil Steril 71,1014-1018.

Plachot M (1992) Cytogenetic analysis of oocytes and embryos. Ann Acad Med Singapore 21,538-544.

Polak de Fried E, Blanco L, Lancuba S and Asch RH (1993) Improvement of clinical pregnancy rate and implantation rate of in-vitro fertilizationembryo transfer patients by using methylprednisone. Hum Reprod 8, $393-395$.

Rink K, Delacretaz G, Salathe RP, Senn A, Nocera D and Germond M (1994) $1.48 \mu \mathrm{m}$ diode laser microdissection of the zona pellucida of mouse zygotes. SPIE 2134A,412-422.

Rink K, Delacretaz G, Salathe RP, Senn A, Nocera D, Germond M, De Grandi P and Fakan S (1996) Non-contact microdrilling of mouse zona pellucida with an objective-delivered 1.48-microns diode laser. Lasers Surg Med 18,52-62

Sallam HN, Sadek SS and Agameya AF (2003) Assisted hatching-a metaanalysis of randomized controlled trials. J Assist Reprod Genet 20, $332-342$.

Schachter M, Raziel A, Friedler S, Strassburger D, Bern O and Ron-El R (2001) Monozygotic twinning after assisted reproductive techniques: a phenomenon independent of micromanipulation. Hum Reprod 16, 1264-1269.

Schieve LA, Meikle SF, Peterson HB, Jeng G, Burnett NM and Wilcox LS (2000) Does assisted hatching pose a risk for monozygotic twinning in pregnancies conceived through in vitro fertilization? Fertil Steril 74, $288-294$.

Senn A, Vozzi C, Chanson A, De Grandi P and Germond M (2000) Prospective randomized study of two cryopreservation policies avoiding embryo selection: the pronucleate stage leads to a higher cumulative delivery rate than the early cleavage stage. Fertil Steril 74,946-952.

Sheen TC, Chen SR, Au HK, Chien YY, Wu KY and Tzeng CR (2001) Herniated blastomere following chemically assisted hatching may result in monozygotic twins. Fertil Steril 75,442-444.

Sills ES, Moomjy M, Zaninovic N, Veeck LL, McGee M, Palermo GD and Rosenwaks Z (2000) Human zona pellucida micromanipulation and monozygotic twinning frequency after IVF. Hum Reprod 15,890-895.

Stein A, Rufas O, Amit S, Avrech O, Pinkas H, Ovadia J and Fisch B (1995) Assisted hatching by partial zona dissection of human pre-embryos in patients with recurrent implantation failure after in vitro fertilization. Fertil Steril 63,838-841.

Tao J and Tamis R (1997) Application of assisted hatching for 2-day-old, frozen-thawed embryo transfer in a poor-prognosis population. $\mathrm{J}$ Assist Reprod Genet 14,128-130.

Yakin K, Balaban B and Urman B (2003) Risks of monochorionic pregnancies after assisted hatching? Fertil Steril 79,1044-1046.

Submitted on April 2, 2004; accepted on June 2, 2004 